\title{
Les brouillons « entre les langues » de Raoul
}

\section{Hausmann}

Dada plurilingue, entre montage et optophonie

\section{Agathe Mareuge}

\section{(2) OpenEdition}

\section{Journals}

Édition électronique

URL : http://journals.openedition.org/genesis/2877

DOI : 10.4000/genesis.2877

ISSN : 2268-1590

Éditeur :

Presses universitaires de Paris Sorbonne (PUPS), Société internationale de génétique artistique littéraire et scientifique (SIGALES)

\section{Édition imprimée}

Date de publication : 4 juin 2018

Pagination : 103-114

ISBN : 979-10-231-0604-6

ISSN : $1167-5101$

\section{Référence électronique}

Agathe Mareuge, «Les brouillons « entre les langues » de Raoul Hausmann », Genesis [En ligne], 46 | 2018, mis en ligne le 01 juin 2019, consulté le 06 septembre 2019. URL : http:// journals.openedition.org/genesis/2877; DOI : 10.4000/genesis.2877 


\title{
Les brouillons «entre les langues» de Raoul Hausmann Dada plurilingue, entre montage et optophonie
}

\author{
Agathe Mareuge
}

\section{Raoul Hausmann et le plurilinguisme dada}

En 1918, dans le sillage de la révolte dadaïste entreprise deux ans plus tôt au Cabaret Voltaire, à Zurich, se forme le «Club Dada» à Berlin, autour de Raoul Hausmann, Richard Huelsenbeck, Johannes Baader, Walter Mehring, Jefim Golyscheff, John Heartfield et Georges Grosz ${ }^{1}$. Parti de Suisse, avant de gagner Paris, New York et d'autres villes, Dada essaime ainsi en Allemagne, et particulièrement à Berlin, principalement durant les années 1918-1921 - le point culminant étant atteint avec la «Première Foire Internationale Dada» durant l'été 1920. Pourquoi «Dada»? Parce que ce mot ne signifie rien, et tout à la fois; selon la légende, le nom de «Dada» fut trouvé en ouvrant au hasard un dictionnaire. À ce sujet, Hugo Ball écrit dans son journal :

En français cela signifie «cheval de bois». En allemand «va te faire, au revoir, à la prochaine». En roumain «oui en effet, vous avez raison, c'est ça, d'accord, vraiment, on s'en occupe», etc. C'est un mot international. Seulement un mot et ce mot comme mouvement. Très facile à comprendre ${ }^{2}$

Comme l'indique déjà son nom, le mouvement Dada est intrinsèquement plurilingue, car, né pendant et à cause de la Première Guerre mondiale, il réunit, d'abord en territoire neutre (la Suisse), des artistes et poètes de toutes nationalités (allemande, française, suisse, autrichienne, roumaine, italienne...) qui entendent opposer à la boucherie de la guerre les possibilités de l'art et du langage, en recourant d'abord au non-sens, à la subversion et à la provocation. Les poètes dada écrivent presque tous en plusieurs langues, soit qu'ils les maîtrisent d'emblée (ainsi l'Alsacien Arp, qui écrit en allemand et en français 3 ), soit qu'ils ajoutent à leur langue maternelle celle(s) du ou des pays d'adoption ou d'exil (français, anglais principalement), avec une réticence grandissante à l'égard de la langue allemande, qui deviendra celle

Genesis 46, 2018 du régime national-socialiste. Les productions dada seront considérées par les nazis comme «art dégénéré».

Dada exprime en effet une double révolte, à la fois artistique et culturelle, qui se fonde sur une critique radicale des langues et des langages artistiques de l'époque. D'emblée, les dadaïstes contestent les conceptions classiques de l'art et de la littérature : ils en déconstruisent les formes, les matériaux et les pratiques, renouvellent les conceptions traditionnelles de l'œuvre d'art et de la notion d'auteur, combattent l'institution littéraire et artistique telle qu'elle existait alors. Dada s'attaque en outre à la société bourgeoise européenne, tenue pour responsable du conflit mondial, et, au-delà, entend mettre à l'épreuve les fondements de la civilisation et de la rationalité occidentales.

Raoul Hausmann, né à Vienne en 1886, fut un acteur majeur de ce mouvement. Il toucha à tous les domaines, brouillant les frontières établies entre les genres artistiques : poèmes phonétiques et visuels, photomontages, assemblages, sans oublier une importante production théorique et critique, dont un grand nombre de manifestes satiriques dirigés notamment contre la République de Weimar. À partir de 1933, Raoul Hausmann est contraint à l'exil : il émigre

1. Hannah Höch, Wieland Herzfelde (frère de John Heartfield) joueront également un rôle important, et Kurt Schwitters s'associera à Hausmann pour une tournée dada internationale.

2. Hugo Ball, Premier manifeste dada, Zurich, 14 juillet 1916, trad. française de Sabine Wolf dans Hugo Ball, Dada à Zurich - Le mot et l'image (1915-1916), Dijon, Les presses du réel, 2006, p. 9.

3. J'ai consacré deux articles au plurilinguisme de Jean Hans Arp. Agathe Mareuge, "Arp et la traduction: "l'acte décisif d'un poète" ", dans Le Texte et l'Idée, CEGIL, Presses universitaires de Nancy, $\mathrm{n}^{\circ} 28,2014$, p. 165-176, et «"D'un monde lointain, étrange, étranger..." : Arp traducteur et la langue maternelle du poète», dans Charlotte Baker et Delphine Grass (dir.), «The Multilingual Spaces of French and Francophone Writing», Nottingham French Studies, n 56.2, 2017, p. 177-187. 
d'abord à Ibiza, puis, à partir de 1936, lorsqu'éclate la guerre civile espagnole, il fuit en Suisse, en Tchécoslovaquie et à Paris avant de se réfugier finalement dans le Limousin, d'abord à Peyrat-le-Château, puis à Limoges. Là, jusqu'à sa mort en 1971, il maintient pendant près de trois décennies une triple activité de création poétique (poésie phonétique et optophonétique), artistique (peinture, collage, photographie) et théorique (notamment histoire de Dada et du néodadaïsme). Son œuvre tardive est caractérisée par le désir de « devenir auteur français ${ }^{4}$ » tout en continuant d'écrire en allemand, et d'être donc publié non seulement en Allemagne, mais aussi en France. Il multiplie alors à la fois l'écriture directement en français et la traduction de textes poéticocritiques, traduction qu'il effectue lui-même en raison des refus essuyés auprès de traducteurs.

Le Musée départemental d'art contemporain de Rochechouart dans le Limousin conserve, dans les archives du Fonds Raoul Hausmann 5 , un nombre important de brouillons et de lettres de l'artiste, qui documentent la genèse de ses textes et le processus de création plurilingue au cœur de son œuvre 6 . Il apparaît que si le plurilinguisme de Raoul Hausmann est d'abord subi, résultant de l'exil, il sera rapidement revendiqué par le poète qui en fait un outil essentiel de sa création poétique et de sa production théorique, toutes deux placées sous le signe de Dada. Deux exemples l'attestent particulièrement : l'ouvrage théorique Courrier Dada 7 et le «roman» (ou anti-roman) Hylé8.

Le premier exemple, celui de Courrier Dada, correspond à un ensemble d'essais dont la genèse est longue et complexe : la rédaction, qui s'organise autour des manifestes dada de Raoul Hausmann datant des années 1918-1921, débute en allemand autour de 1939; elle se poursuit jusqu'en 1956; cependant, à partir de 1945, Raoul Hausmann traduit en parallèle ces textes en français. Il en poursuivra l'écriture directement en français jusqu'en 1958, date de la parution de Courrier Dada à Paris. Une version allemande remaniée et abrégée paraît en 1972 sous le titre Am Anfang war Dada ${ }^{9}$, mais la version allemande complète Kurier Dada correspondant précisément à Courrier Dada, conservée à Rochechouart, reste encore inédite.

Dans le cas du roman Hylé, on a affaire selon Raoul Hausmann à un «genre de roman en forme de rêve ${ }^{10}$ ». On peut considérer ce texte comme un «work in progress» (d'après Hélène Thiérard ${ }^{11}$ ) dont la genèse s'étend sur plus de trois décennies, de 1926 à la fin des années 1950, voire au début des années 1960, d'après la correspondance avec les éditeurs. Dans ce roman double, la première partie, Hylé I, encore inédite à ce jour, se rapporte principalement aux années allemandes de Raoul Hausmann (1926-1933); la seconde partie, Hylé II (publiée en 2006), est essentiellement dévolue aux années d'exil à Ibiza (1933-1936) : en adéquation avec le contenu largement autobiographique, l'écriture polyphonique devient alors en outre plurilingue 12 .

L'étude des différents textes et brouillons relatifs à ces deux ensembles montre que la pratique de l'écriture

4. Lettre de Raoul Hausmann à Eugène Jolas, 11 mai 1945, reproduite dans Courrier Dada, nouvelle édition établie, augmentée et annotée par Marc Dachy, Paris, Allia, 1992, p. 172. La formule est reprise par Adelheid Koch-Didier dans «"Devenir auteur français". Raoul Hausmann», dans Henri Béhar, Catherine Dufour, Dada Circuit total, Lausanne, L'Âge d'homme, coll. «Les Dossiers H», 2005, p. 582-589.

5. Je remercie Chantal François-Texier, responsable du Fonds Raoul Hausmann, et Annabelle Ténèze, conservatrice du Musée départemental d'art contemporain de Rochechouart, de m'avoir donné accès à ces archives.

6. On se reportera à l'inventaire raisonné établi par Adelheid Koch-Didier au Fonds Raoul Hausmann, "Je suis l'homme de 5000 paroles et de 10000 formes ». Écrits de Raoul Hausmann et documents annexes répertoriés, Inventaire Raisonné, avec la collaboration de Stefan Schwar, Rochechouart, 1997.

7. Raoul Hausmann, Courrier Dada, Paris, Le Terrain Vague, 1958.

8. Raoul Hausmann, Hyle. Ein Traumsein in Spanien, München, Belleville, 2006. Le livre, y compris la postface d'Adelheid Koch-Didier, a été traduit en français par Hélène Thiérard: Raoul Hausmann, Hylé. État de rêve en Espagne, Dijon, Les presses du réel, 2013.

9. Raoul Hausmann, Am Anfang war Dada, Gießen, Anabas Verlag, [1972] 1992.

10. Cité par Adelheid Koch-Didier dans «Nachwort», Hylé, op. cit., p. 334, trad. française d'Hélène Thiérard dans Hylé, op. cit., p. 368.

11. Voir Hélène Thiérard, Hylé I et Hylé II de Raoul Hausmann : des ensembles autobiographiques en mouvement, thèse de doctorat, université de la Sorbonne Nouvelle/université d'Osnabrück, 2016.

12. Il existe un troisième exemple de processus créatif plurilingue dans l'œuvre tardive de Raoul Hausmann : sa correspondance avec Kurt Schwitters en 1946-1947 pour leur projet de revue PIN, qui entendait retracer de manière créative ce qu'avaient été les années dada pour les deux artistes. Schwitters vit lui aussi en exil jusqu'à sa mort en 1948, en Grande-Bretagne. Rejetant la langue allemande pervertie par les nazis, les deux artistes correspondent en anglais et intègrent toutes les langues apprises en exil (dont le norvégien pour Schwitters). Ils ne se reverront jamais et le projet n'aboutira pas - faute d'éditeur intéressé - si bien qu'il n'existe que sous forme épistolaire, prenant forme au fil des lettres. $P I N$ a été publié en 1962 par Jasia Reichardt, nièce d'un ami de 
plurilingue, à laquelle se livre alors le «dadasophe» (comme Hausmann se nomme lui-même avec une pointe d'humour, insistant par là sur la dimension réflexive de sa pratique artistique dada), est paradigmatique de sa poétique, particulièrement de sa poétique tardive 13 . Ce plurilinguisme révèle deux caractéristiques essentielles de sa création.

Premièrement, l'écriture plurilingue et l'un de ses avatars, l'autotraduction, apparaissent comme l'une des manifestations de sa pratique du montage, du collage, de l'assemblage de fragments - en l'occurrence, en différentes langues -, que Raoul Hausmann exerce dès Dada Berlin, surtout dans le domaine plastique, avec de nombreux photomontages (fig. 1) ou encore avec l'assemblage de sa célèbre Tête mécanique ou L'Esprit de notre temps de 1919, aujourd'hui conservée au Centre Pompidou (fig. 2).

Deuxièmement, le plurilinguisme est un ressort de l'écriture «optophonétique ${ }^{14}$ », c'est-à-dire une écriture qui transformerait en matériau sonore les impressions visuelles et corporelles perçues par le poète. Il s'agit de parvenir, par l'usage de plusieurs langues, à un degré de sensorialité supplémentaire; pour cela, le poète explore la matérialité sonore, visuelle - des différents idiomes, indépendamment de leur charge sémantique, ce que permet particulièrement le travail avec des langues étrangères. Nous examinerons successivement ces deux aspects.

\section{Des montages entre les langues}

Le procédé du montage et de l'assemblage est ce qui frappe d'emblée lorsque l'on considère l'ensemble des textes relatifs à Courrier Dada, et lorsque l'on se penche sur l'exercice de traduction et de rédaction conjointe en allemand et en français auquel se livre Hausmann dès 1945. Le projet de cet ouvrage est de raconter ce que fut Dada Berlin au moyen de documents historiques : les manifestes écrits ou co-écrits dans les années 1918-1921. Ces textes qui relèvent d'une strate temporelle ancienne sont insérés et montés dans un texte explicatif plus tardif (1939-1956), qui prend lui-même différentes formes. Dans les premières versions allemandes restées inédites (les manifestes étant originellement écrits en allemand, c'est en allemand que Raoul Hausmann les présente), il s'agit de brefs textes introductifs, conclusifs ou de transition, de nature essayistique, parfois à la tonalité polémique, qui visent à expliciter les manifestes en les resituant dans le contexte de l'époque. La structure du texte est donc déjà d'emblée composite. À cette première hétérogénéité vient s'ajouter celle introduite par les va-et-vient entre deux langues, l'allemand et le français.

Ne trouvant pas d'éditeur en Allemagne, Hausmann entreprend à partir de 1945 de traduire son texte en français, et d'en poursuivre la rédaction directement en français tout en continuant à travailler au manuscrit allemand. Or la traduction française est à l'origine d'un changement de forme : les chapitres de l'ouvrage se transforment en «lettres 15 » adressées à une certaine Mademoiselle Maud, amie (réelle) de Hausmann, d'où le titre : Courrier Dada à une jeune femme d'aujourd'hui. 10 et une lettres. Seules cinq de ces lettres en français nous sont parvenues (deux ont été publiées dans des revues en 194616, trois sont inédites) : elles sont toutes conservées aujourd'hui dans le fonds Raoul Hausmann de Rochechouart. À la lecture des brouillons de ces lettres - adressées donc à un destinataire réel, mais d'emblée destinées à être publiées -, on observe que si

Schwitters : la publication, après une introduction retraçant la genèse de PIN, présente l'ensemble des textes prévus par Hausmann et Schwitters, reconstituant ce qu'aurait pu devenir ce projet inachevé. Voir Raoul Hausmann, Kurt Schwitters, PIN and the Story of PIN, dir. Jasia Reichardt, London, Gaberbocchus Press, 1962, repr. Gießen, Anabas Verlag, 1986; et en français Isabelle Ewig (présentation, traduction et annotation), «Retour vers le futur : correspondance Schwitters-Hausmann 19461947 », Cahiers du Musée national d'art moderne, n 76, été 2001.

13. Sur l'œuvre de Raoul Hausmann à Ibiza et sur l'esthétique tardive de Raoul Hausmann historien de l'art, voir Cécile Bargues, Raoul Hausmann après Dada, Bruxelles, Mardaga, 2015; sur Raoul Hausmann, poète expérimental et ses relations avec ses contemporains, voir Timothy Benson, Hanne Bergius, Ina Blom (dir.), Raoul Hausmann et les avantgardes, Dijon, Les presses du réel, 2015.

14. Hausmann publie son premier texte relatif à sa théorie optophonétique en 1922 dans la revue hongroise MA éditée par Lajos Kassák et László Moholy-Nagy : «Optofonetika», dans MA 8, n 1, 1922. Une version française paraîtra en 1969 en revue et en 1970 dans un ouvrage de Hausmann : «Optophonétique», De Tafelronde 14, n 2, 1969, p. 62-66 et Raoul Hausmann, La Sensorialité excentrique, Cambridge, Ormiston, 1970, p. 6-10.

15. Ces chapitres se présentent sous forme de lettres mais nous ne pouvons pas affirmer avec certitude qu'elles n'ont pas été effectivement envoyées à leur destinataire.

16. Raoul Hausmann, «Courrier Dada à une jeune femme d'aujourd'hui », Arts-Lettres 1, n 6, 1946, p. 293-294 et «Dada est plus que Dada», ibid., p. 295-299. 


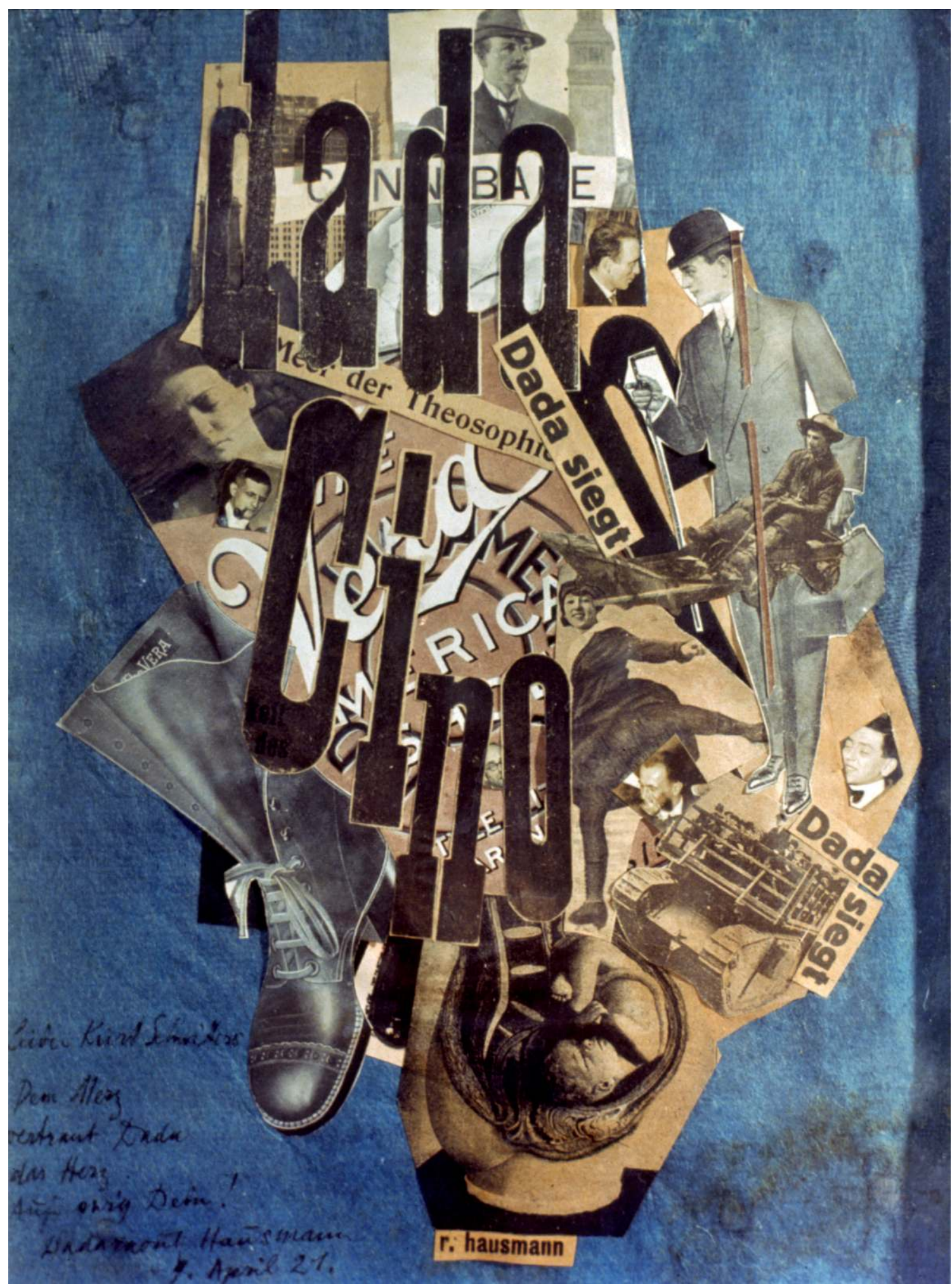

Fig. 1 : Raoul Hausmann, Dada cino, 1920

collage et photomontage sur papier, 31, x $22,5 \mathrm{~cm}$, coll. part. (c) D.R. 


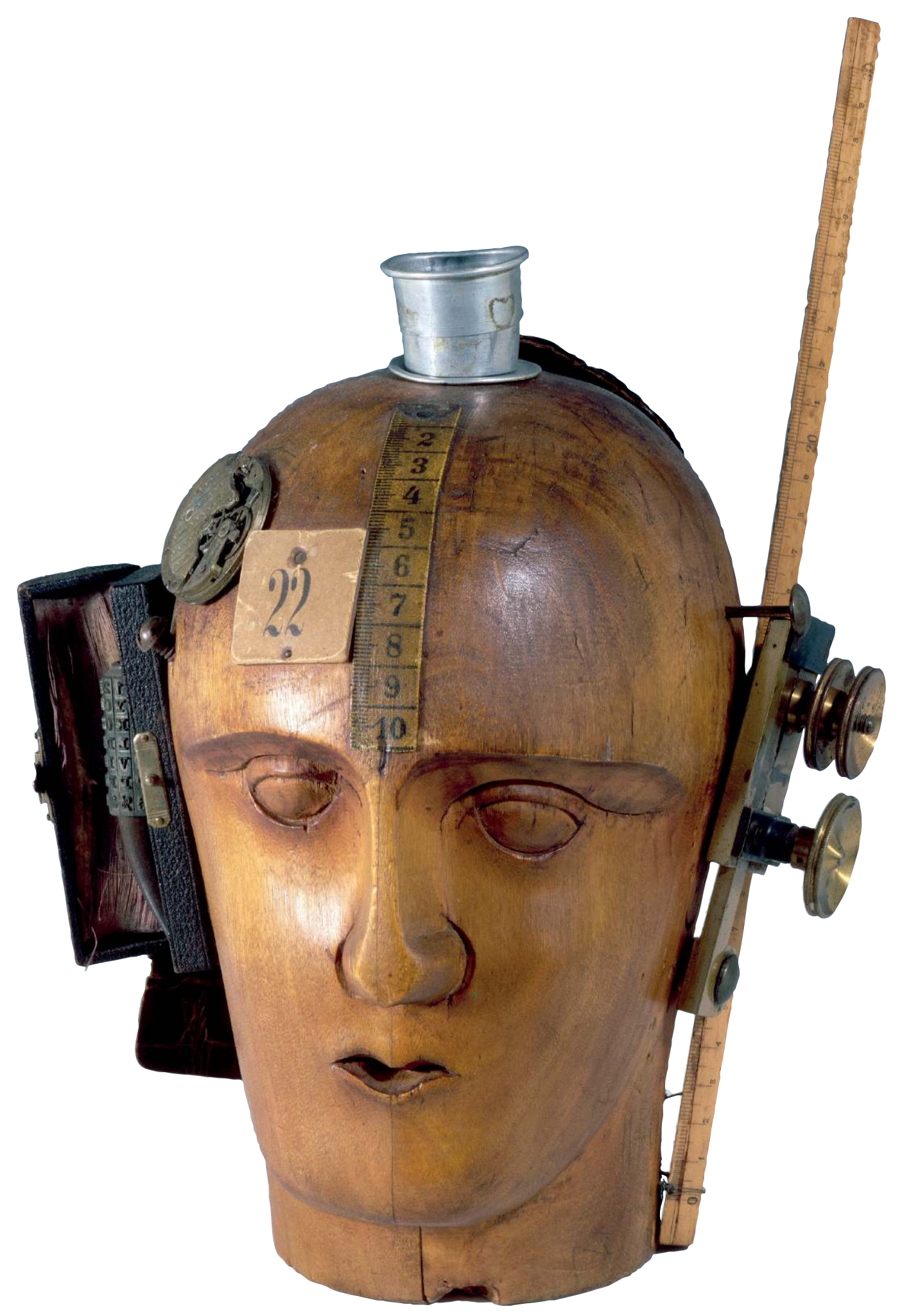

Fig. 2 : Raoul Hausmann, Mechanischer Kopf/Tête mécanique (L'esprit de notre temps), 1919, assemblage, $32,5 \times 21 \times 20 \mathrm{~cm}$, Paris, Musée national d'art moderne - Centre de création industrielle, Centre Georges-Pompidou

Photo (C) Centre Pompidou, MNAM-CCI, Dist. RMN-Grand Palais/image Centre Pompidou, MNAM-CCI - @ ADAGP, Paris 


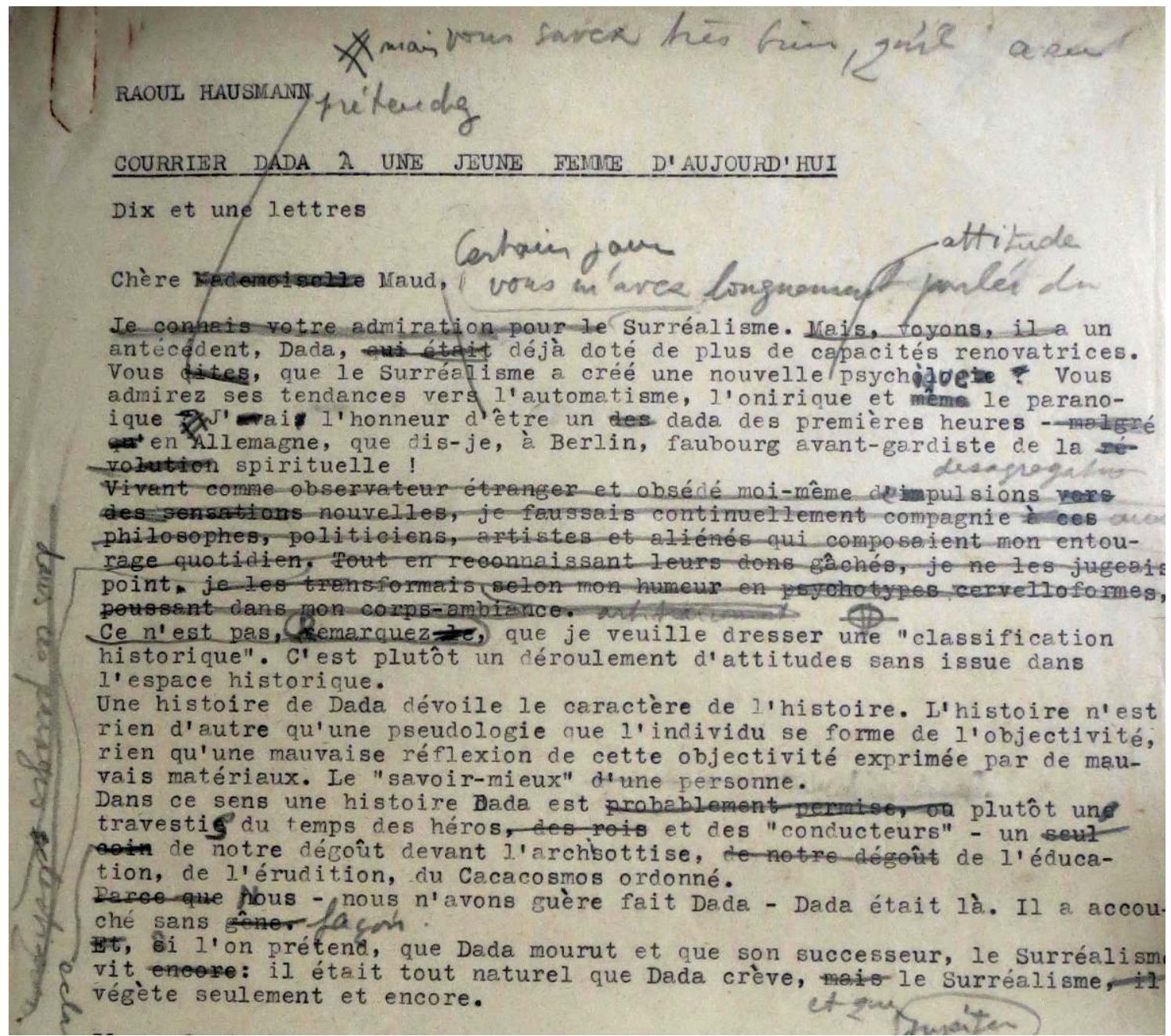

Fig. 3 : Raoul Hausmann, Courrier Dada à une jeune fille d'aujourd'hui, première page, tapuscrit conservé au Musée départemental d'art contemporain de Rochechouart (cote A.II.1.2/tx-C)

«Chère Mademoiselle Maud,

Je connais votre admiration pour le Certain jour vous m'avez longuement parlé du Surréalisme. Mais, voyons, il a Mais vous savez très bien qu'il a eu un antécédent, Dada, qui était déjà doté de plus de capacités rénovatrices. Vous dites prétendez que le Surréalisme a créé une nouvelle psychølogie attitude psychique ? Vous admirez ces tendances vers l'automatisme, l'onirique et même le paranoïque. J'avais ai l'honneur d'être un des dada des premières heures - malgré qu'en Allemagne, que dis-je, à Berlin, faubourg avant-gardiste de la révolution désagrégation spirituelle !

Vivant comme observateur étranger et obsédé moi-même d'impulsions vers des sensations nouvelles, je faussais continuellement compagnie à ces philosophes, politiciens, artistes et aliénés qui composaient mon entourage quotidien. Tout en reconnaissant leurs dons gâchés, je ne les jugeai point, je les transformais selon mon humeur en psychotypes cervelloformes poussant dans mon corps-ambiance artificiellement.

Ce n'est pas, [Remarquez-łe], que je veuille dresser une «classification historique». C'est plutôt un déroulement d'attitudes sans issue dans l'espace historique.

Une histoire de Dada dévoile le caractère de l'histoire. [...]*»

(*) Fonds Raoul Hausmann, Musée départemental d'Art contemporain de Rochechouart. Notre transcription. 
l'essentiel du texte et de son argumentation demeurent très proches de l'original allemand, la mise en forme diffère sensiblement; la présence d'un destinataire modifie non seulement le ton adopté, qui se fait professoral, mais aussi le mode de réflexion et d'expression. Hausmann choisit le mode de la dispute, bien vivante - comme du temps de Dada - en même temps que celui de l'explication.

Il s'agit ici (fig. 3) de l'incipit de la première «lettre», qui correspond grosso modo au texte allemand intitulé «Dada ist mehr als Dada» et contient le manifeste du même nom, daté de 1921. L'extrait ci-dessus est ajouté par rapport au texte allemand, qui commence seulement au quatrième paragraphe ( «Eine Geschichte DADA enthüllt den Charakter der Geschichte $/ / \ll$ Une histoire de DADA dévoile le caractère de toute Histoire», selon la version française publiée en 1958, qui a finalement renoncé à la forme épistolaire). Sur ce brouillon conservé à Rochechouart, qui montre un état du texte qui ne sera en fin de compte pas maintenu par l'auteur lors de la publication ${ }^{17}$, on peut lire les passages biffés et modifiés par Hausmann, c'est-à-dire l'argumentation en train de se faire, la pensée en train de se construire. Et l'on note l'importance du contexte français au moment de la traduction, qui détermine cet ajout par rapport à la version allemande : la prédominance du surréalisme dans le Paris de l'après-guerre, donc le besoin d'affirmer l'antériorité de Dada, ainsi que la nécessité de préciser à un auditoire peu familier ce que fut le Berlin de l'après-Première Guerre mondiale, et les querelles entre artistes. En traduisant, Raoul Hausmann complète et adapte donc son propos au contexte culturel français en même temps qu'il joue avec les formes, prouvant que la notion d'œuvre, déjà mise à mal par Dada, ne constitue plus un cadre fixe de référence mais peut être sans cesse redéfinie. La pratique de l'autotraduction souligne le procédé du montage qui constituait déjà, au niveau intralinguistique, le principe même de composition de Courrier Dada / Kurier Dada.

De même, la création du roman Hylé se présente comme un assemblage de multiples unités textuelles (le tout compte plus de mille pages), à l'image des Trois chaises évoquées dans le livre et qui font partie des quelques fragments autotraduits en français par Hausmann : «Oh, quel ouvrage, quel assemblage, quel chevronnage : trois chaises. Le menuisier a placé trois chaises dans l'espace vide de la Sala haute. Étant en bois et en corde, une fonction a pris forme ${ }^{18}$.» Hylé se présente en effet comme un ensemble composite, hybride, un assemblage de fragments - ce qui ressort dans les extraits traduits en français, que l'auteur a pour la plupart dotés de titres («L'image», «Triquadre», «Molgrano», «L'ordre», etc.), comme si ces fragments pouvaient aussi bien fonctionner de manière autonome qu'être intégrés à un ouvrage plus vaste. Cet assemblage déconstruisant le genre romanesque traditionnel est caractérisé par son inachèvement de principe : au fil des années, des demandes des éditeurs et des tentatives de traduction, Hausmann ne cesse de couper, d'insérer, de réagencer son texte. La présence de blancs, d'interruptions dans le matériau textuel rend aussi visible l'acte d'écriture, par l'entremêlement de passages narratifs, de rêves, de poèmes, de réflexions mythologiques, ethnographiques, philosophiques, poétologiques, ou encore par la juxtaposition des niveaux linguistiques et stylistiques (le dadasophe se targue d'employer « 50 styles ${ }^{19} »$ ), avec une audace à côté de laquelle Alfred Döblin passe pour «conventionnel», d'après le lecteur des Éditions Claassen qui envisagent un temps de publier Hylé20. Ici aussi donc, la conception classique de l'œuvre est subvertie - et la filiation à cet égard serait plutôt à chercher du côté des premiers romantiques allemands, qui ont fait du fragment leur forme privilégiée :

Le caractère intrinsèquement processuel du projet Hylé tend ainsi à la subversion de l'opposition entre achevé et inachevé - entre texte et avant-texte. [...] Ainsi, non seulement Hylé I et Hylé II sont parcourus par de nombreuses frontières internes du fait du montage, mais certaines de ces frontières internes (entre les différentes unités) constituent également des frontières externes (avec d'autres projets d'écriture). Le rapport entre le dedans et le dehors des ensembles textuels est donc subverti par le biais de leur forme plurielle. Qui plus

17. Malgré le maintien du titre Courrier Dada, Raoul Hausmann renonce finalement à la forme épistolaire au moment de la publication, tout en conservant une grande partie des textes qui en sont issus. Subsistent en outre l'emploi de la première personne du singulier et des adresses au lecteur (du type : «Lisez...»).

18. En allemand : «Oh welch Gewerke, Gefüge, Gesperre: drei Stühle hat der Tischler in den leeren Raum der hohen Sala gestellt. Aus Holz und Seilen; eine Funktion nahm Form. » Fonds RH, Rochechouart.

19. Lettre de Raoul Hausmann à Renée Sulzbach, 15 juin 1966, fonds RH, Rochechouart, citée dans Adelheid Koch-Didier, «Nachwort», dans Raoul Hausmann, Hyle, op. cit., p. 321-346.

20. Ibid., p. 325. 
est, la pratique de l'autotraduction en français dans Hylé II fait vaciller le statut respectif du texte original et du texte traduit 21 .

L'une des caractéristiques essentielles de ce texte mosaïque réside dans ce que Adelheid Koch-Didier a appelé sa « décentralité langagière 22 » : au sein même de la version originale allemande se côtoient le yiddish (la femme et la compagne d'Hausmann sont juives), le français, l'anglais, l'espagnol, l'ibizenco... Par cette écriture plurilingue, le poète entend libérer le langage du carcan grammatical et syntaxique qui l'emprisonne dans chaque langue. Les néologismes et constructions translingues au sein d'une même phrase (qui commence dans une langue et se poursuit dans une ou plusieurs autres) sont récurrents dans la version allemande première. Ils résultent d'abord des nombreux dialogues, souvent rapportés au style indirect ou indirect libre, entre le personnage principal, Gal (double de l'auteur), et les personnes qu'il fréquente sur l'île, véritable microcosme babélien : les habitants parlant majoritairement le dialecte ibizenco, mais aussi les nombreux immigrés parlant anglais, français, allemand, yiddish, etc. Cette logique d'affranchissement des normes monolingues conduit, dans un double processus, à s'approprier des langues étrangères tout en rendant étrangère la langue maternelle allemande.

Le phénomène continue et s'amplifie encore davantage dans les fragments recréés par Hausmann en français, une langue qui lui est moins familière (et où subsistent d'ailleurs des erreurs ou approximations). C'est ce qu'on peut observer dans l'extrait suivant 23 (fig. 4), qui est une véritable recréation, puisqu'il n'y a pas d'équivalent exact dans la version allemande - on retrouve seulement certains motifs et l'écriture comme flux de pensées et de sensations. Le protagoniste, Gal, laisse dériver ses pensées tandis qu'il marche, portant une cruche d'eau.

On le voit, l'écriture du texte progresse par associations de pensées engendrées par la déambulation dans le paysage d'Ibiza ${ }^{24}$, dans lequel un certain nombre de réalités sont désignées par le vocable correspondant dans le dialecte local (une variante du catalan), suggérant ainsi une identité entre l'expérience sensorielle faite à Ibiza et la langue dans laquelle cette expérience est faite (ainsi, «herbes» d'abord écrit en français est corrigé en «hierbas»). Mais les intrusions de la langue maternelle sont autorisées dans le monologue intérieur («Was ist der Mensch, er ist wie Gras»), de même que les associations sonores, par homophonie : le vide, «das Hohle» en allemand, appelle «the hollow» en anglais; le «cantaro », la cruche, entraîne «le chant à eau », puis la «hanche».

\section{L'écriture optophonétique et la matière des langues}

Le jeu entre les langues, qu'il prenne la forme d'une écriture plurilingue ou de l'autotraduction-recréation, reflète et incarne l'attention portée à la matière du langage. Le titre du «mythe autobiographique» composé par Hausmann (l'expression est de lui ${ }^{25}$ ) est à cet égard parlant : hylé désigne en grec ancien la matière, le matériel comme opposé au spirituel (eidos). Pour Hausmann, qui puise à la fois dans la philosophie grecque et dans la pensée phénoménologique, hylé est la matière primordiale, aussi bien celle dont est faite la vie, que celle dont est fait le langage, unis dans le rêve - le sous-titre, «état de rêve», est indissociable du titre. Le projet de Hausmann consiste précisément à unir les deux, en imposant au lecteur un autre rapport à la langue. Dans une lettre à Hausmann, l'artiste et poète concret Carlfriedrich Claus note d'ailleurs que ce texte doit être lu à voix haute pour prendre toute son ampleur («laut sprechen»), et il parle de la parole 26 de Hausmann plus que de son texte. Il faut préciser que les expériences menées par Hausmann sur le langage depuis 1918 portent essentiellement sur sa

21. Hélène Thiérard, Hylé I et Hylé II de Raoul Hausmann : des ensembles autobiographiques en mouvement, op. cit., p. 439.

22. «sprachliche Dezentralität», Adelheid Koch-Didier, «Nachwort», dans Raoul Haumann, Hyle, op. cit., p. 333, trad. française d'Hélène Thiérard dans Raoul Hausmann, Hylé, op. cit., p. 366.

23. «C'est presque comme les poules...», brouillon, Fonds RH, Rochechouart. Le marquage en caractères gras n'est pas de Raoul Hausmann mais de nous; il vise à faire ressortir tous les passages en langues autres que le français, langue principale de l'extrait.

24. On sait que Raoul Hausmann a lu, entre autres, Ulysses de Joyce en traduction allemande en 1928.

25. Cité par Adelheid Koch-Didier dans «Nachwort», Hyle, op. cit., p. 334, trad. française d'Hélène Thiérard dans Raoul Hausmann, Hylé, op. cit., p. 368.

26. Lettre de Carlfriedrich Claus à Raoul Hausmann, 17 juillet 1960, fonds RH, Rochechouart. 


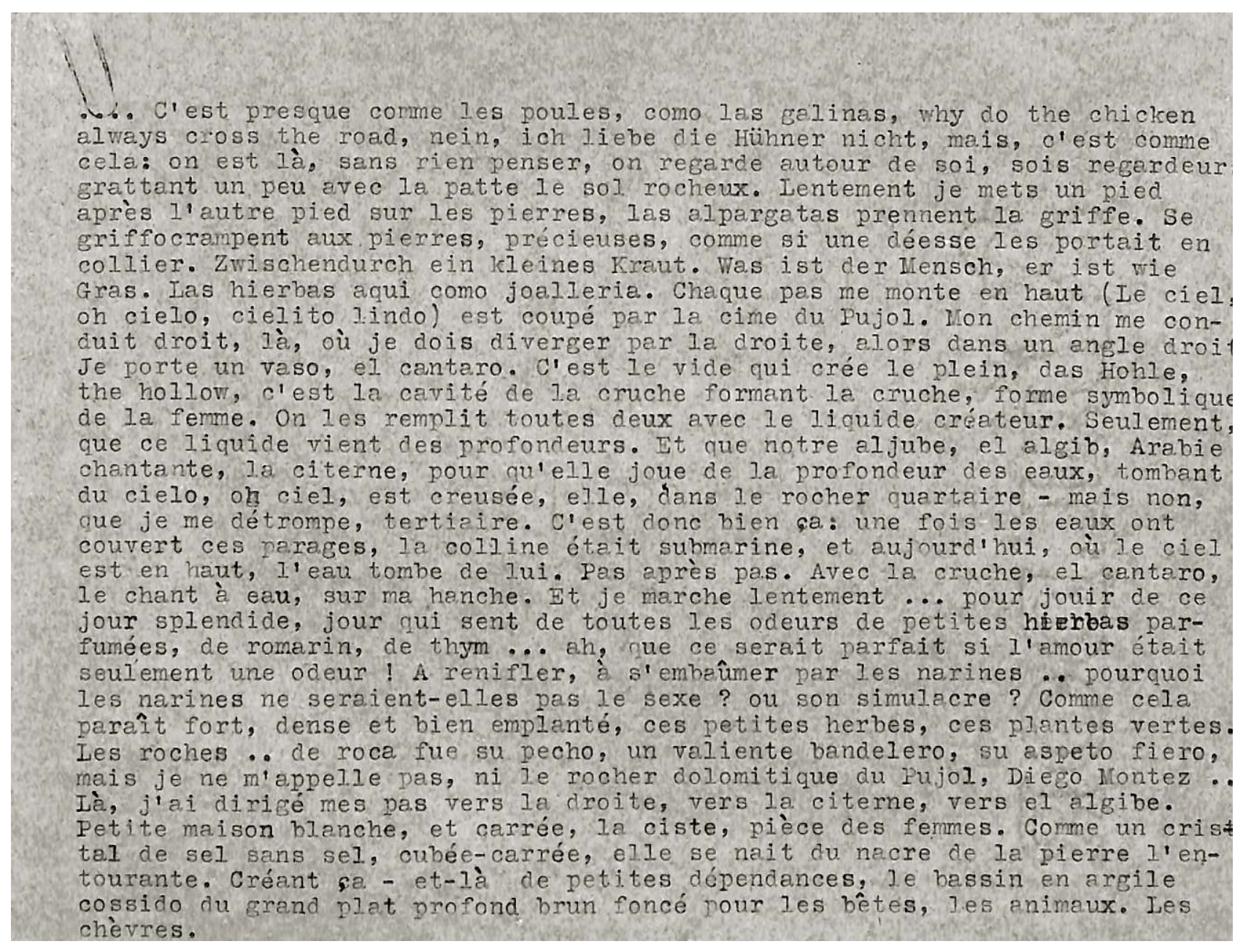

Fig. 4 : Raoul Hausmann, «C'est presque comme les poules...»,

fragment de Hylé, tapuscrit conservé au Musée départemental d'art contemporain de Rochechouart (cote C.I.2.5/sd-04)

... C'est presque comme les poules, como las galinas, why do the chicken always cross the road, nein, ich liebe die Hühner nicht, mais, c'est comme cela : on est là, sans rien penser, on regarde autour de soi, sois regardeur : grattant un peu avec la patte le sol rocheux. Lentement je mets un pied après l'autre pied sur les pierres, las alpargatas prennent la griffe. Se griffocrampent aux pierres, précieuses, comme si une déesse les portait en collier. Zwischendurch ein kleines Kraut. Was ist der Mensch, er ist wie Gras. Las hierbas aqui come joalleria. Chaque pas me monte en haut (Le ciel, oh cielo, cielito lindo) est coupé par la cime du Pujol. Mon chemin me conduit droit, là, où je dois diverger par la droite, alors dans un angle droit. Je porte un vaso, el cantaro. C'est le vide qui crée le plein, das Hohle, the hollow, c'est la cavité de la cruche formant la cruche, forme symbolique de la femme. On les remplit toutes deux avec le liquide créateur. Seulement, que ce liquide vient des profondeurs. Et que notre aljube, el algib, Arabie chantante, la citerne, pour qu'elle joue de la profondeur des eaux, tombant du cielo, oh ciel, est creusée, elle, dans le rocher quartaire - mais non, que je me détrompe, tertiaire. C'est donc bien ça : une fois les eaux ont couvert ces parages, la colline était submarine, et aujourd'hui, où le ciel est en haut, l'eau tombe de lui. Pas après pas. Avec la cruche, el cantaro, le chant à eau, sur ma hanche. Et je marche lentement... pour jouir de ce jour splendide, jour qui sent de toutes les odeurs de petites heshierbas parfumées, de romarin, de thym... ah, que ce serait parfait si l'amour était seulement une odeur! À renifler, à s'embaûmer par les narines... pourquoi les narines ne seraient-elles pas le sexe? ou son simulacre ? Comme cela paraît fort, dense et bien emplanté, ces petites herbes, ces plantes vertes. Les roches... de roca fue su pecho, un valiente bandelero, su aspeto fiero, mais je ne m'appelle pas, ni le rocher dolomitique du Pujol, Diego Montez... 


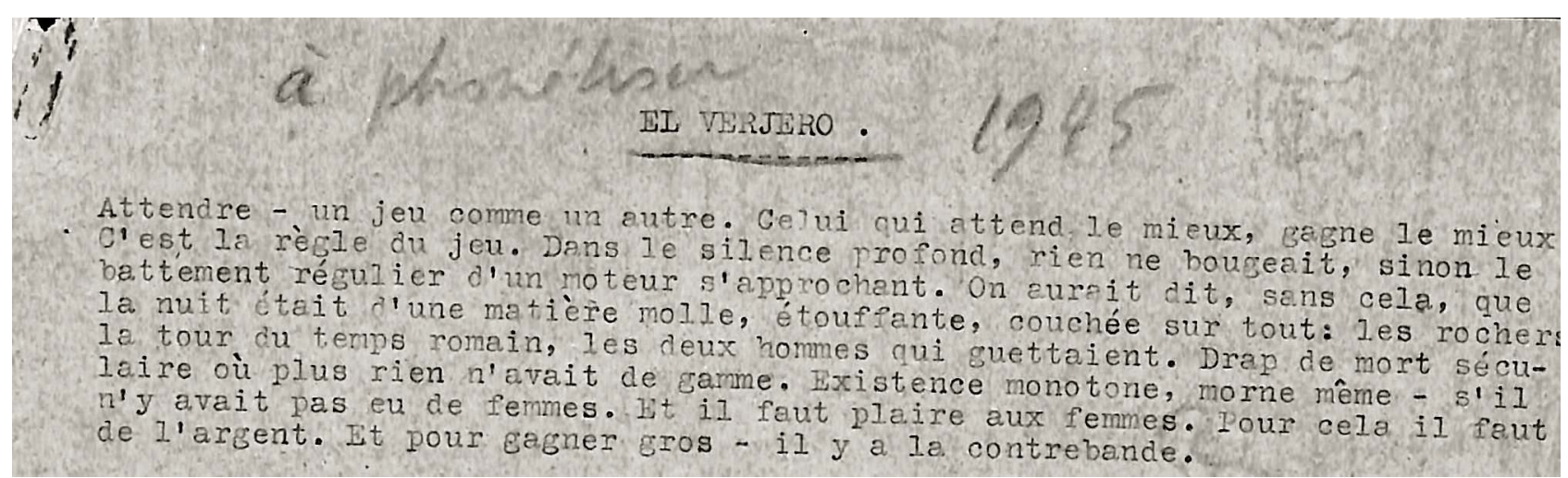

Fig. 5 : Raoul Hausmann, «El Verjero», fragment de Hylé,

tapuscrit conservé au Musée départemental d'art contemporain de Rochechouart (cote C.I.2.5/45)

dimension sonore; il a d'ailleurs à cœur, lorsqu'il écrit l'histoire du Lautgedicht (poème phonétique) après 1945, de réaffirmer son propre rôle dans l'invention de la poésie sonore, et notamment dans l'invention de la suite de sons «fmsbw» sur laquelle se fonde la Ursonate ou «Sonate en sons primitifs » de Kurt Schwitters. Le brouillon d'un fragment français de Hylé intitulé El Verjero ${ }^{27}$ porte du reste la mention manuscrite «à phonétiser» (fig. 5).

Parmi les extraits que le poète a autotraduits ou recréés en français figure un fragment qu'il a intitulé «Molgrano», Molgrane, «mille grains », c'est-à-dire la grenade, fruit du grenadier (qu'on appelle aussi «pomme punique»), doté dans Hylé d'une fonction à la fois mythique et mythologique, et assimilé à Tanit-Astarté, déesse phénicienne et carthaginoise de la fertilité. Dans cet extrait, ce qui parle ou plutôt «voit-parle» (siehtspricht) pour reprendre l'expression de Hausmann, c'est «la langue du signe» (des Zeichens Sprache), et ce qui importe est de brouiller les oppositions entre sujet et objet, intérieur et extérieur, ouïe et vision : «Ich nähere mich Baum, Dir. [...] Siehtspricht in mir.» est traduit par «Je m'approche arbre - de toi. [...] Regarde parle en moi 28 .»

Que montre le travail de transposition de l'allemand au français? Tout d'abord, on relève l'indétermination du terme «arbre», qui peut être lu comme apposé à «m'» (dans le sens de «moi qui suis arbre») ou bien comme une adresse à «toi », indiquant une fusion entre le sujet et l'arbre, plus explicite qu'en allemand où elle n'est suggérée que par la virgule qui rapproche l'arbre de la première plutôt que de la deuxième personne; on note ensuite que «sieht» est traduit par «regarde» (actif) et non pas simplement «voit» (qui serait plus passif). C'est la «voix de l'image» (des Bildes Ton) qui parle ainsi. Un autre élément significatif de ce fragment et des recréations françaises en général tient à l'usage du tiret qui permet à Hausmann d'unir deux impressions, deux perceptions et de rendre le mot composé allemand (zusammengesetzte Form), en créant des néologismes à l'envi : «noir-obscur» (dunkelschwarz), «auréole-croissante brille luisante» (Wachstum glänzt leuchtend aureal), «allongé-enmietté » [sic] (langhingebröselt), «source-racine-jaillit» (Wurzelspringquell), «pareil à des lances-éclats soufflent les feuilles-syllabes dans les brasbranches autour» (Glanzlanzen gleich wehn Blättersilben in der Zweig-Arme umher).

Il apparaît dans cet exemple que l'ambition de l'artiste et poète dans Hylé est bien de réunir, dans le mot, à la fois son, vision et parole. Hylé, cet «état de rêve / état-rêve en Espagne» (Traumsein in Spanien) explore une écriture qui soit l'expression de toutes les impressions corporelles et sensorielles. Il faut rappeler qu'Hausmann est l'inventeur d'un dispositif qu'il a même fait breveter, l'optophone, qui transforme en ondes sonores les ondes visuelles. On pourrait ainsi dire, en reprenant les termes de l'auteur, que ce qui se joue dans Hylé est la mise en place d'une écriture non pas

27. Fonds RH, Rochechouart.

28. «Molgrano», Fonds RH, Rochechouart. 
tant phonétique qu'optophonétique. Précisons d'ailleurs que Hausmann fut un grand photographe et que son séjour à Ibiza notamment a donné lieu à une très importante production photographique, complémentaire de l'écriture du livre29 . À Ibiza, un lieu en retrait du monde où Raoul Hausmann cherche à retrouver une autre forme de vie, proche de la nature, faite d'observations quasi anthropologiques et ethnographiques, le poète fait l'expérience de l'élasticité du temps, ayant perdu avec l'exil les repères de sa vie d'avant. Le temps à la fois dure et semble suspendu. Comme l'a également montré l'extrait précédent, Hausmann aspire dans Hylé à retrouver une unité primordiale et archaïque, qui serait pour lui la fusion de l'esprit et de la matière au moyen du rêve ou du mythe, conçu non pas comme extérieur à la vie, mais bien comme la vie même.

\section{Conclusion : quadrils et triquadres entre les langues}

Comme nous avons été obligés de vivre dans différentes parties d'Europe, nous sommes capables de parler et d'écrire dans plusieurs langues, tels : l'allemand, l'anglais, le français, le norvégien et l'espagnol. Ainsi nous désirons appeler nos poèmes : quadril, pour ceux écrits par Hausmann (allfrananglespagnol) et triquadra, pour ceux écrits par Schwitters (allangnorvégien). Il n'est toutefois pas nécessaire qu'un poème contienne toutes les langues parlées par son auteur, ils peuvent être créés dans une seule langue. [...] Quadril et Triquadra comme forme poétique doivent être aussi parfaits que la pierre taillée, aussi parfait qu'un quadrilatère 30 .
Triquadre - variante de «triquadra»-, c'est le titre dont Hausmann a doté l'un des fragments de Hylé qu'il a autotraduit en français, reprenant le nom de cette «forme poétique» inventée par lui dans sa correspondance avec Schwitters pour le projet de revue PIN, formulé en anglais. Ces propos peuvent aussi s'appliquer à Hylé et montrent bien que le plurilinguisme de Hausmann est devenu, à partir de son exil, une caractéristique intrinsèque de son écriture, à la fois lorsqu'elle est effectivement plurilingue et intègre des fragments en langue étrangère, et, au-delà, même au sein des textes écrits dans une seule langue. L'allemand ou le français sont désormais nourris du plurilinguisme vécu par l'auteur et en sont profondément marqués, du fait que toutes ses langues - maternelle ou acquises plus tard - semblent être devenues «étrangères » à leur auteur. Ainsi, les brouillons de Raoul Hausmann révèlent que son écriture tardive entre les langues se combine avec des procédés tels que le montage, l'assemblage, ou encore avec l'optophonie, qui prennent leur source dans le contexte plurilingue des années dada. L'expérience de l'exil leur donne un nouveau relief.

29. Voir le catalogue de l'exposition récente conçue par Cécile Bargues et présentée au Point du Jour (Cherbourg) et au Jeu de Paume (Paris) : Cécile Bargues, Raoul Haussman, Photographies 1927-1936, Cherbourg : Le Point du Jour, Paris : Jeu de Paume, Rochechouart : Musée départemental d'art contemporain, 2017.

30. Lettre de Raoul Hausmann à Kurt Schwitters, 23 août 1946, repr. dans «Dossier PIN» établi par Isabelle Ewig, dans Kurt Schwitters, catalogue d'exposition, Paris, Éditions du Centre Pompidou, 1994, p. 348-353, ici p. 348. 
Agathe Mareuge, agrégée d'allemand, est maître de conférences en Études germaniques et médiation culturelle à l'université Paris-Sorbonne. Elle poursuit à l'université de Zurich un projet de recherche consacré à la réinvention et à l'héritage de Dada après 1945 (post-doc SNF, dir. Sandro Zanetti). Sa thèse (à paraître aux Presses du Réel) était consacrée à l'œuvre poétique tardive de l'artiste et poète Jean Hans Arp.

agathe.mareuge@sorbonne-universite.fr

Résumés

\section{Les brouillons «entre les langues» de Raoul Hausmann}

Cette contribution prend appui sur les brouillons plurilingues de l'écrivain et artiste Raoul Hausmann, «dadasophe» autoproclamé, à son «anti-roman» Hylé et son recueil d'essais Courrier Dada. Ces brouillons sont conservés dans les archives du fonds Raoul Hausmann du Musée de Rochechouart, dans le Limousin, où Hausmann vécut en exil à partir de 1939. Ils montrent que l'autotraduction à laquelle il se livre alors est paradigmatique de sa poétique. Premièrement, l'(auto)traduction apparaît comme un nouvel avatar des techniques de montage, de collage et d'assemblage, que Raoul Hausmann pratiqua dès Dada Berlin (1918-1920). Deuxièmement, la traduction fut pour Hausmann l'occasion de mettre en œuvre une écriture «optophonétique», c'est-à-dire qui transforme en sons, en matériau sonore les impressions visuelles et corporelles perçues par le poète. Les brouillons documentent ainsi le processus de création «entre les langues » qui est au cœur de l'œuvre haussmannienne.

This article studies the multilingual drafts of the "anti-novel" Hyle and the collection of essays Courrier Dada by the self-proclaimed "dadasopher" artist and writer Raoul Hausmann. These drafts are held in the Raoul Hausmann Collection of the Rochechouard Museum in the Limousin region where Hausmann lived in exile from 1939. They show that the self-translation to which he then had recourse is paradigmatic of his poetics. In the first place, self-translation appears as a new avatar of the montage, pasting and mounting techniques practiced by Hausmann as early as the Berlin Dada period (1918-1920). Secondly, translation was for Haumman the opportunity to implement "optophonetic" writing, i.e. that transforms into sounds visual and physical impressions experienced by the poet. These drafts thus document the creative process "between languages" at the heart of Haussmann's work.

Ausgangspunkt dieses Aufsatzes sind die mehrsprachigen Textentwürfe und -varianten zu dem „Antiroman“ Hyle und der Essaysammlung Courrier Dada des Schriftstellers und Künstlers Raoul Hausmann, der sich selbst den „Dadasophen“ nannte. Diese Texte liegen im Raoul-Hausmann-Archiv des Museums in Rochechouart in der Region Limousin, in der Hausmann nach 1939 im Exil lebte. Sie belegen, dass Hausmanns Selbstübersetzungen für seine Poetik paradigmatisch sind. Erstens kann die (Selbst-)Übersetzung als eine neue Variante der Montage-, Collage- und Assemblage-Techniken betrachtet werden, die Raoul Hausmann seit den Jahren von Dada Berlin (1918-1920) benutzte. Zweitens verwendete Hausmann beim Übersetzen ein sogenanntes ,optophonetisches" Schreibverfahren, das die vom Dichter erfahrenen visuellen und leiblichen Eindrücke in Laute und Tonmaterial verwandelt. Die Textentwürfe dokumentieren somit den Schaffensprozess Hausmanns und zeigen, wie er ,zwischen den Sprachen“ arbeitet.
Esta contribución se basa en los borradores multilingües del escritor y artista Raoul Hausmann ("dadásofo" autoproclamado) de su "antinovela" Hylé y su recopilación de ensayos Courrier Dada. Estos borradores, que están guardados en los archivos del Fondo Raoul Hausmann del Museo de Rochechouart, en la región de Lemosín, donde Hausmann vivió exiliado a partir de 1939, ponen en evidencia que la autotraducción que el escritor practica es paradigmática de su poética. Primero, la (auto)traducción aparece como un nuevo avatar de las técnicas de montaje, collage y armado, que Raoul Hausmann pone en práctica desde los tiempos de Dada Berlín (1918-1920). Segundo, la traducción constituye para Hausmann la ocasión de poner en funcionamiento una escritura "optofonética", es decir, que convierte en sonidos, en material sonoro, las impresiones visuales y corporales percibidas por el poeta. Estos borradores dan testimonio así del proceso de creación "entre las lenguas" que está en el centro de la obra hausmanniana.

Esta contribuição parte dos rascunhos multilingues do escritor e artista Raoul Hausmann, auto-proclamado "dadasophe", nomeadamente o "anti-romance" Hylé e a colecção de ensaios Courrier Dada. Esses rascunhos conservam-se no Arquivo Raoul Hausmann, do Museu de Rochechouart, no Limousin, onde Hausmann viveu exilado a partir de 1939. A auto-tradução a que ele se dedica é paradigmática da sua poética. Em primeiro lugar, a (auto)tradução revela-se como um novo avatar das técnicas de montagem, colagem e junção, que Raoul Hausmann praticava desde Dada Berlin (1918-1920). Em segundo lugar, a tradução deu a Hausmann a oportunidade de desenvolver uma escrita "optofonética", que transforma as impressões visuais e corpóreas captadas pelo poeta em som, em material acústico. Os rascunhos mostram, assim, um processo criativo „entre línguas“, que está no cerne da obra do autor.

L'articolo si basa sui manoscritti preparatori multilingue dell" "anti-romanzo" Hylé e della raccolta di saggi Courrier Dada dello scrittore e artista Raoul Hausmann, "dadasofo" autoproclamato. Questi manoscritti - conservati negli archivi del Fondo Raoul Hausmann del Museo di Rochechouart, nel Limousin, dove Hausmann visse in esilio dal 1939 - dimostrano come l'autotraduzione sia paradigmatica della sua poetica. In primo luogo, l'(auto)traduzione può essere considerata come un nuovo avatar delle tecniche di collage, montaggio e assemblaggio, praticate da Raoul Hausmann fin dagli anni di Dada Berlin (1918-1920). In secondo luogo, la traduzione rappresenta per Hausmann l'occasione di realizzare una scrittura "optofonetica", che trasforma cioè le impressioni visive e corporee percepite dal poeta in suoni, in materiale sonoro. I manoscritti preparatori documentano così il processo di creazione "fra le lingue", al centro dell'opera hausmanniana. 